\title{
A Change of the Gravitational Interaction on the Galactic Distances
}

\section{Stabnikov PA and Babailov SP*}

Nikolaev Institute of Inorganic Chemistry, Siberian Branch of Russian Academy of Sciences, 630090 Novosibirsk, Russia

${ }^{*}$ Corresponding author: Babailov SP, Nikolaev Institute of Inorganic Chemistry, Siberian Branch of Russian Academy of Sciences, 630090 Novosibirsk, Russia, Tel: +7(383)- 3308957; E-mail: babajlov@niic.nsc.ru

Rec date: Jul 05, 2015; Acc date: Aug 14, 2015; Pub date: Aug 18, 2015

Copyright: (c) 2015 Stabnikov PA, et al. This is an open-access article distributed under the terms of the Creative Commons Attribution License, which permits unrestricted use, distribution, and reproduction in any medium, provided the original author and source are credited.

\begin{abstract}
In order to explain anomalous velocities of star rotation in galaxies, some researchers propose corrections to Newton's dynamics. In contrast to this opinion, we suppose that it is possible to correct the potential of gravity interaction. The modulus of the force of interaction between the bodies may be written as: $F=E x p\left(-R^{2} / \alpha\right) y M m / R^{2}+$ $\left(1-\operatorname{Exp}\left(-R^{2} / \alpha\right)\right) \delta M m / R$, где $\alpha \sim 4 \times 10^{5}(a u)^{-2}, \delta \sim 2.7 \times 10^{-31} \mathrm{~N} \mathrm{~m} \mathrm{~kg}^{-2}$. This correction allows one to conserve the force of attraction between the bodies within the Solar System proportional to $1 / \mathrm{R}^{2}$, while for longer interstellar distances it will be proportional to 1/R. It provides a good description both for the motion of planets in the Solar System and for the motions of stars and galaxies. Within the framework of the proposed approach, there is no necessity to introduce the idea of dark matter. According to the analysis of the proposed interaction with the help of the virial theorem, it does not contradict basic notions on the motion of stars and planets; in particular, it does not contradict the established accelerated expansion of the Universe.
\end{abstract}

Keywords: Astronomy; Dark matter; Virial theorem; Gravity interaction; Kepler's laws

\section{Introduction}

Classical gravitational interaction between material bodies characterized by the force is proportional to $1 / \mathrm{R}^{2}$ works well within the Solar System. It is still accepted that the gravitational interaction can be spread in non-amended form up to boundaries of our Universe. The virial theory for classical gravitational interaction between masses gives equation $2 \mathrm{~T}=-\mathrm{U}[1]$, where $\mathrm{T}$ is the average kinetic energy and $\mathrm{U}$ is average potential energy. This relation is well fulfilled for the motion of planets in the Solar System. However, investigation of the motion of stars in galaxies and the motion of galaxies with respect to each other resulted in the accumulation of facts that required some supplements or changes to classical notions. For instance, Figure 1 shows how the velocities of stars rotation around the center of the Galaxy change.

This is a generalized figure characteristic of the majority of spiral galaxies. Near the center up to a definite critical region $\mathrm{R}_{\mathrm{c}} \sim 8 \mathrm{\kappa pc}$, a linear increase in the velocities of star rotation around the center is observed. This region of the central part of the galaxy with approximately constant density of stellar matter is called Bulge. Beyond $R_{c}$ the density of the stellar matter in the galaxy decreases, which is confirmed by astronomical observations. In this situation, the Velocities of star rotation should decrease according to Kepler's laws, similarly to the velocities of planet rotation in the Solar System (curve disk, Figure 1). This would be in complete agreement with the conclusions of virial theory. However, it follows from experiments that the velocities of star rotation at distances larger than $R_{c}$ remain nearly constant till the edge of the Galaxy. It follows from this fact that the longer is the distance from $\mathrm{R}_{\mathrm{c}}$ to a star, the stronger its motion deviates from Kepler's laws and conclusions of virial theory.

As far as the observed velocities of the motions of galaxies and estimations of their masses are concerned, substantial differences are observed, too. To determine the mass of a galaxy, there are two

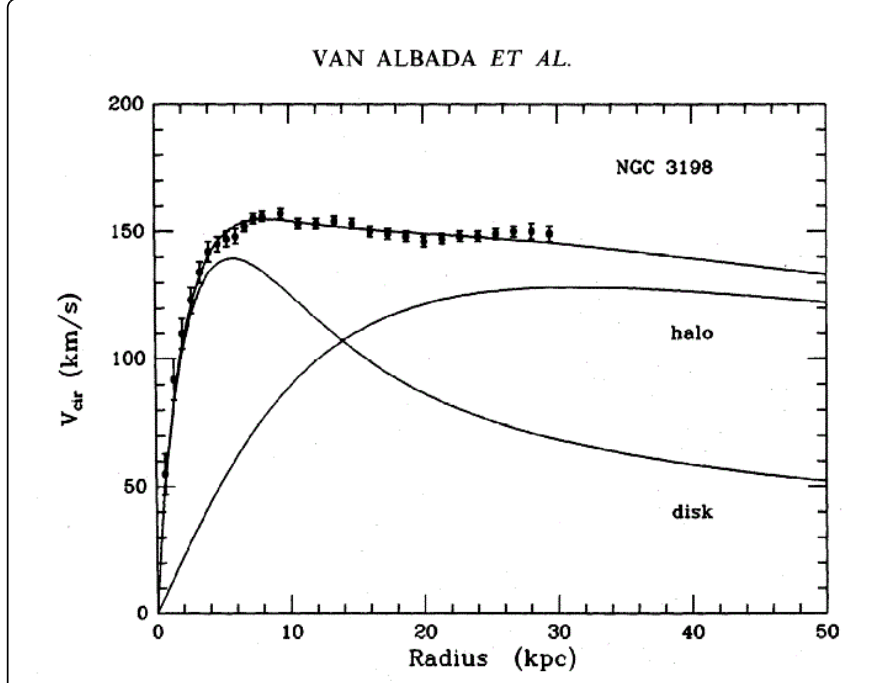

Figure 1: The dependence of the stars' rotation speed and of dark matter distribution from distance to the center of the galaxy (http:// bustard.phys.nd.edu/Phys171/lectures/dm.html).

independent methods. One of them is based on the fact that the masses of the accumulations of galaxies and separate star systems are directly connected with their brightness. The higher is brightness, the larger is the mass. The other method is dynamics, it is based on the conclusions of virial theory, namely on the dependence between the masses of accumulations and the velocities of their own motions. These velocities and the distances between these accumulations can be established rather reliably, so it is not difficult to calculate the masses of the accumulations. Comparison between the results obtained using these methods showed that the masses of accumulations determined from their brightness are much smaller than the masses determined using the dynamic method. It turned out that the difference was 
Page 2 of 4

several ten and hundred times, which cannot be attributed to measurement errors. This discrepancy can be corrected using three ways:

1. To introduce invisible dark matter (DM)

2. To correct Newton's dynamics

3. To correct the classical potential of gravity at large distances, this is proposed in the present work.

Now we will consider these approaches in more detail.

1. Some researchers [2-4] proposed to introduce invisible dark matter (DM) to correct the situation. From the viewpoint of virial theorem, this will increase the potential energy of attraction in any system, which is to recover the relation $2 \mathrm{~T}=-\mathrm{U}$. This will be so only in the case if DM does not contribute into the kinetic energy, that is, DM must not move at all. In addition, to keep the velocities of star rotation around the center of galaxy constant at distances longer than $R_{c}$, it is necessary that the density of DM should permanently increase from the center to periphery curve halo, Figure 1 . Unusual properties of DM follow from this fact: visible matter must be attracted to DM, while the latter is to repel the visible matter because the density of DM in galaxy center is minimal (Figure 1). In other words, DM should be immobile (possess no kinetic energy) and possess unusual attraction-repulsion properties. In addition, it is not clear up to what limit the density of DM will increase at the periphery of galaxy. Because of this, the introduction of DM is not very successful method of solving the problem. Besides, to explain all the anomalies in the velocities of motion, for stars and for galaxies, some researchers claimed that our world by more than $95 \%$ would consist of DM and dark energy (dark energy is introduced to explain accelerated expansion of the Universe) [5]. Because of this, new approach that would exclude the introduction of non-observable DM remains to be attractive for researchers. To confirm this idea, Vera Rubin said: "If I could have my pick, I would like to learn that Newton's laws must be modified in order to correctly describe gravitational interactions at large distances. That's more appealing than a universe filled with a new kind of sub-nuclear particle."

2. Alternative approaches to correct the situation include a number of methods aimed at modifications of Newton's dynamics (MOND (Modified Newtonian Dynamics) [6], TeVeS (Tensor-Vector-Scalar Gravity) [7], NGT (Nonsymmetric Gravitational Theory) [8], "dark fluid" (Chaplygin gas) [9], double metric tensor [10], etc.). However, these approaches are rather complicated and allow one to explain only the constancy of the velocities of star rotation at the periphery of galaxies. The application of the virial theorem is not discussed within these approaches.

3. We propose to correct the classical potential of gravitational interaction at long distances similarly to the manner in which this was done for Van der Waals interaction between atoms and molecules. The foundations of this approach include the consideration of interactions between bodies as a sum of several terms; each of these terms makes a decisive contribution within a limited distance range. For instance, for the interactions between micro particles, the zone of repulsion and several zones of attraction are distinguished. For the astronomical distances we propose two terms of interaction; one of them, similarly for classical gravity, is valid inside the Solar System $\left(\sim 1 / R^{2}\right)$, while the other works for longer distances $(\sim 1 / R)$. It should be noted that the attempts to modify gravity potential are known in astronomy. For example, in order to explain the anomalous motion of the Moon, as long ago as in 1745 Clairaut proposed to write Newton's law as $F=\gamma M_{1} M_{2}\left(1 / R^{2}+\alpha / R^{4}\right)$. Later (in 1752) Clairaut concluded that the classical law is quite sufficient to explain the motion of the Moon with all its anomalies. Nevertheless, the idea of Clairaut in various mathematical implementations appeared several times in the history of astronomy, in particular to explain the shift of the perihelion of Mercury. However, Einstein demonstrated in 1915 that within the General Theory of Relativity (GTR) a deviation from the classical trajectory of planetary motion should be observed near a massive body. The calculated values of the shift of the perihelion of Mercury precisely coincided with astronomical observations, which became also an experimental confirmation of GTR.

The approach proposed by us will allow us to explain both the anomalies in the motions of starts at the periphery of galaxies and the anomalies of the motions of galaxies. In addition, the application of virial theorem to the interaction between material bodies with the forces proportional to $1 / \mathrm{R}$ leads to another relation between the kinetic and potential energies, which will better describe the energy of interacting masses at super long distances.

\section{Comparison of Two Potentials}

The interaction between bodies for two potentials-classical gravitational potential, which is proportional to $1 / \mathrm{R}^{2}$, and the new one proposed in the present work, which is proportional to $1 / \mathrm{R}$ - is presented in the (Table 1) Here $\mathrm{m}$ is mass of the planet or star; $M$ is mass of the central part; $\gamma$ is the gravitational constant; $\delta$ is constant of new interaction.

\begin{tabular}{|l|l|l|}
\hline Interaction mode & $\mathrm{CGI}-\mathrm{\gamma}$ & $\mathrm{NI}-\delta$ \\
\hline Dependence on distance & $1 / \mathrm{R}^{2}$ & $1 / \mathrm{R}$ \\
\hline Expression for the force & $\mathrm{F}=\mathrm{\gamma Mm} / \mathrm{R}^{2}$ & $\mathrm{~F}=\delta \mathrm{Mm} / \mathrm{R}$ \\
\hline Equality in a circular orbit & $\mathrm{m} \mathrm{V}^{2} / \mathrm{R}=\mathrm{\gamma Mm} / \mathrm{R}^{2}$ & $\mathrm{mV} / \mathrm{R}=\delta \mathrm{Mm} / \mathrm{R}$ \\
\hline Expression for the square of the velocity & $\mathrm{V}^{2}=\mathrm{yM} / \mathrm{R}$ & $\mathrm{V}^{2}=\delta \mathrm{M}$ \\
\hline Expression for the rotational invariants & $\mathrm{V}^{2} \mathrm{R}=\mathrm{\gamma M}$ & $\mathrm{V}^{2}=\delta \mathrm{M}$ \\
\hline
\end{tabular}

Table 1: Comparison of two interactions: the classical gravitational (CGI), proportional $1 / \mathrm{R}^{2}$ and the new interaction (NI) proposed in this paper, which is proportional $1 / \mathrm{R}$.

As it follows from Table, for the bodies with interaction force proportional to $1 / \mathrm{R}^{2}$, the velocity of their rotation around a massive center is Const/R, which is observed in the Solar System. For bodies interacting with a force proportional to $1 / R$, the velocity of their 
rotation around a massive center is Const, which is observed for the rotation of stars at the periphery of galaxies. A simple conclusion may be drawn from these facts: the forces of the interaction of stars with the center of galaxy are proportional to $1 / R$. So, we need a potential the interaction force for which would be proportional to $1 / \mathrm{R}^{2}$ within the Solar System and to $1 / \mathrm{R}$ for interstellar distances. Techniques for superposition of two different contributions from interaction within one potential have been developed long ago for Van der Waals interaction between atoms and molecules [11]. Relying on these data, we propose the expression for the modulus of attraction force:

$$
\mathrm{F}=\operatorname{Exp}\left(-\mathrm{R}^{2} / \alpha\right) \gamma \mathrm{Mm} / \mathrm{R}^{2}+\left(1-\operatorname{Exp}\left(-\mathrm{R}^{2} / \alpha\right) \delta \mathrm{Mm} / \mathrm{R}\right.
$$

Here $\alpha$ is some constant. Below we will omit the term "modulus for simplicity.

Within the present work, we did not intend to explain the physical sense of expression (1). We only may assume that this kind of interaction can be linked in some manner with the finite light speed or with the features of the structure of spate-time at super long distances.

Since the motion of stars at the periphery of galaxies deviates from Kepler's laws, we decided to check whether there are small deviations in the motion of planets at the periphery of the Solar System. For this purpose, we chose the most reliable and complete data on the parameters of the orbits of planets in the Solar System: the Oxford Illustrated Encyclopedia [12] and Internet. On the basis of these data, we plotted the dependence of rotation invariant $\left(\mathrm{V}^{2} \mathrm{R}\right)$ on the distance from the Sun (for the Earth, $\mathrm{R}=1$ ). Results are shown in Figure 2.

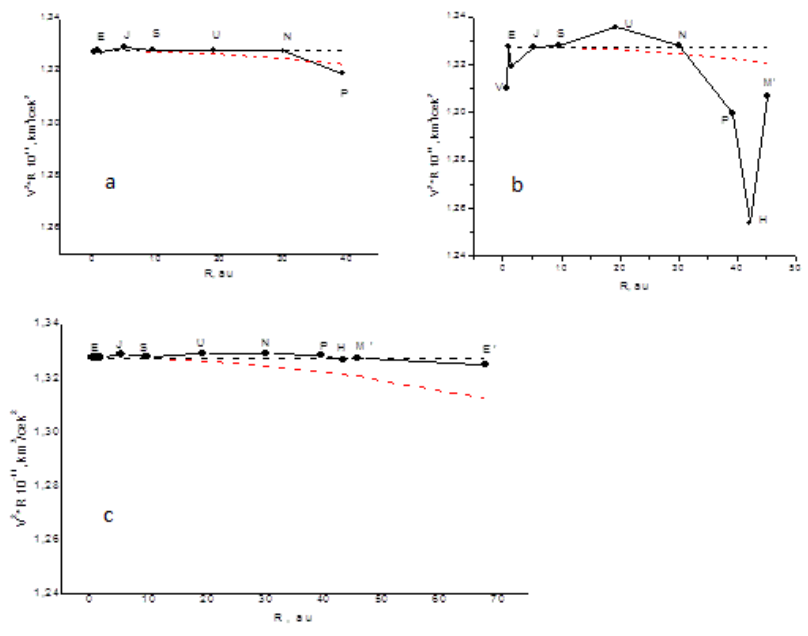

Figure 2: Dependence of the invariant of planetary rotation on the relative distance from the Sun, $2 \mathrm{a}$ - date [12], 2b - date [ru.wikipedia.org/wiki/solar system], 2c - date [http:// www.princeton.edu/ willman/planetary_systems/Sol/]. The red and green dashed lines correspond to the product the value V2R for the Earth on $\operatorname{Exp}\left(-\mathrm{R}^{2} / \alpha\right)$ at $\alpha$ equal $4 \times 10^{5}$. Abbreviations: E, Earth; J, Jupiter; S, Saturn; U, Uranus; N, Neptune; P, Pluto; H, Haumea; M, Makemake; E, Eris.

cIt should be noted that the data available in the literature on small bodies like Haumea, Makemake, Eris and Sedna are unreliable at present. The fact is that they were discovered about 10 years ago. Exact parameters of their orbits and orbital periods have not been established yet. For example, for Eris the orbital radius reported in different publications varies from 37 to $97 \mathrm{au}$. In the internet there are some sites presenting either smoothed data on the parameters of planets and dwarf planets (www.uranian-institute.org/tnpdata.html), or inconsistency data. For example, the site (http://en.wikipedia.org/ wiki/List_of_gravitationally_rounded_objects_of_the_Solar_System) along with $\mathrm{R}$ presents also the revolution periods and the velocities of rotation of the objects in the Solar System but these data are not consistent. It should also be noted that the data on the parameters of planetary orbits are given without errors, which is likely to be connected with not very high accuracy of the values.

Generalizing the available data on the parameters of orbits in the Solar System, we may conclude that there is some trend to a decrease in rotation invariant. We did not detect any data showing that the invariant of rotation increases at the periphery of the Solar System. What may be a reason of a decrease in the invariant of rotation? Relativistic effects in the motion of a planet around the Sun have only insignificant effect on the shift of the perihelion, which is manifested mainly for Mercury. However, these effects have almost no effect on the average speed of revolution. So, relativistic effects cannot explain a decrease in the invariant of rotation of dwarf planets. We suppose that this is the evidence of the degradation of the classical gravity interaction at the periphery of our planetary system.

To characterize this weakening quantitatively, we chose expression $\operatorname{Exp}\left(-R^{2} / a\right)$, where $a=4 \times 10^{5}(a u)^{-2}$. A product of $V^{2} R$ for the Earth and $\operatorname{Exp}\left(-R^{2} / a\right)$ is shown in Figures $2 a-2 c$ as a red dash line. It follows from these plots that this correction of the classical gravity potential will have almost zero effect on the characteristics of planet orbits in our Solar System but will substantially weaken the attraction between stars. The nearest star Proxima of Centaurus is at a distance of $2.68 \times 10^{5} \mathrm{au}$. The force of attraction between the Sun and Proxima of Centaurus, calculated using the classical gravity potential, is equal to $2.06 \times 10^{15} \mathrm{~kg}$. The value of expression $\operatorname{Exp}\left(-\mathrm{R}^{2} / 4 \times 10^{5}\right)$ for this distance is $\sim \operatorname{Exp}\left(-1.8 \times 10^{5}\right) \sim 10^{-7800}$, a very small value; the attraction of the Sun to Proxima of Centaurus multiplied by this value will be close to zero. With this correction, classical gravitational interaction will not affect the interactions of stars with each other and with the center of galaxies, while the new interaction (the force of which is proportional to $1 / \mathrm{R}$ ) will dominate.

So, the expression for the force of interaction between bodies can be written as

$$
\mathrm{M}=\mathrm{V}^{2} \mathrm{R}_{\mathrm{c}} / \gamma
$$

Where, $\mathrm{Rc}$ is the minimal distance (to the center) after which the velocities of star rotation in the galaxy remain constant $(\mathrm{Rc} \sim 8 \mathrm{kpc})$.

Since we assume that the dominant interaction for intragalactic distances becomes the interaction characterized by $\delta$ constant, the corresponding expression for the mass of the centre of galaxy will be:

$$
\mathrm{M}=\mathrm{V}^{2} / \delta \text {. }
$$

Equating 3 to 4 we obtain:

$$
\delta=\gamma / R_{c} \text {. }
$$

For $\mathrm{R}_{\mathrm{c}} \sim 8 \mathrm{kpc} \sim 2.47 \times 10^{20} \mathrm{~m}$, we obtain for the new potential: $\delta \sim$ $6.67 \times 10^{-11} / 2.47 \times 10^{20} \sim 2.70 \times 10^{-31} \mathrm{H} \mathrm{m} \mathrm{kg}^{-2}$

What is the boundary of the range of distances starting from which the dominant interaction will be that characterized by the $\delta$ constant? The equality of the values of $\operatorname{Exp}\left(-R^{2} /\left(10^{6}\right)\right) \gamma / R^{2}$ and $\left(1-\operatorname{Exp}\left(-R^{2} /\right.\right.$ 
$\left.\left(10^{6}\right)\right) \delta / \mathrm{R}$ will occur at a distance of $\sim 2300 \mathrm{au}=1.1 \times 10^{-2} \mathrm{pc}$, which exceeds the distance to heliopause approximately by a factor of 23 (heliopause is $\sim 100 \mathrm{au}$, or $\sim 4.85 \times 10^{-4} \mathrm{pc}[12]$.

We also considered a model expression $\mathrm{F}=\mathrm{mM} / \mathrm{R}^{(1+a)}$, where $\mathrm{a}=4 \times 10^{5} /\left(4 \times 10^{5}+\mathrm{R}^{2}\right)$. However, this approach leads to a drastic decrease in the mass of the galactic center.

\section{Application of Virial Theorem}

According to the proposed expression (1), the force of interaction between bodies consists of two terms: $\operatorname{Exp}\left(-\mathrm{R}^{2} / 4 \times 10^{5}\right) \gamma \mathrm{Mm} / \mathrm{R} 2$ and $(1$ - $\left.\operatorname{Exp}\left(-\mathrm{R}^{2} / 4 \times 10^{5}\right)\right) \delta \mathrm{Mm} / \mathrm{R}$. Additional factors $\operatorname{Exp}\left(-\mathrm{R}^{2} / 4 \times 10^{5}\right)$ and $(1-$ $\left.\operatorname{Exp}\left(-R^{2} / 4 \times 10^{5}\right)\right)$ serve to make the term proportional to $1 / \mathrm{R}^{2}$ work at distances shorter than $2300 \mathrm{au}$, and the term proportional to $1 / \mathrm{R}$ - at substantially longer distances. So we will apply virial theorem to each term separately and only in the distance range where each term dominates. Then the first term will be written as $\gamma \mathrm{Mm} / \mathrm{R}^{2}$, and the second one $\delta \mathrm{Mm} / \mathrm{R}$.

The expression for the kinetic energy of body motion is well known: $\mathrm{T}=\mathrm{mV}^{2} / 2$. The change of the potential energy in the field of a force proportional to $1 / R^{2}$, when passing from radius $R_{1}$ to radius $R_{2}$, is written as a definite integral within the limits $R_{1}$ to $R_{2}: \Delta U=\int F$ $\mathrm{dR}=\int \gamma \mathrm{mM} / \mathrm{R}^{2} \mathrm{dR}=-\gamma 1 \mathrm{mM} / \mathrm{R}_{1}+\gamma 1 \mathrm{mM} / \mathrm{R}_{2}$. Usually an infinite quantity is chosen for $R_{2}$. Then $U=-\gamma 1 \mathrm{mM} / R_{1}$. The relation between the kinetic and potential energy of bodies rotating in the field of forces proportional to $1 / \mathrm{R}^{2}$ is well known: $2 \mathrm{~T}=-\mathrm{U}$ [1]

The change of the potential energy in the field of the force proportional to $1 / R$, passing from radius $R_{1}$ to radius $R_{2}$, is written as a definite integral within the limits $R_{1}$ to $R_{2}: \Delta U=\int F d R=\int \delta m M / R$ $\mathrm{dR}=\delta m M \operatorname{Ln}\left(\mathrm{R}_{1}\right)-\delta m M \operatorname{Ln}\left(\mathrm{R}_{2}\right)$. It is known that the logarithmic function becomes equal to zero for the argument equal to 1 . It is this value that we will choose as one of the limits of integration. This value will be called the galactic universal unit (GUU). With this approach, $\Delta \mathrm{U}=\delta \mathrm{mM} \operatorname{Ln}(\mathrm{R})$ for distances shorter than GUU will be negative, while for distances longer than GUU it will be positive. Negative $U$ values are characteristic of stable systems, while positive ones are characteristic of unstable ones.

To deduce a relation between the kinetic and potential energies of the system in the field of a force which is proportional to $1 / R$, we will write the equality of centrifugal and centripetal forces for a rotating body: $\mathrm{mV}^{2} / \mathrm{R}=\delta \mathrm{mM} / \mathrm{R}$. Тогда $\mathrm{T}=\mathrm{mV}^{2} / 2=\delta \mathrm{mM} / 2=\delta \mathrm{mM} \quad \mathrm{Ln}(\mathrm{R}) /$ $(2 \operatorname{Ln}(R))=\Delta U /(2 \operatorname{Ln}(R))$. Or, therefore:

$$
(2 \operatorname{Ln}(\mathrm{R})) \mathrm{T}=-\mathrm{U}
$$

This is the relation between the kinetic and potential energies for the systems in the field of forces proportional to $1 / R$, which differs from results [1] for systems in the field of forces proportional to $1 / \mathrm{R}^{2}$.

Then we will make an attempt to estimate GUU. It is clear that his value should be larger than the size of the galaxies that are dynamically stable. The radius of our Galaxy is $1.5 \times 10^{1} \mathrm{kpc}$, the radius of galaxy IC $1101-\mathrm{K}=3 \times 10^{2} \mathrm{kpc}$. Because of this, for GUU value we accept $10^{3}$ $\mathrm{kpc}$. According to virial theorem, for distances longer than GUU the potential energy of the systems is positive. This means that, in spite of the fact that the galaxies attract to each other, recession of galaxies is profitable from the point of view of the energy. The longer is the distance to the galaxies, the stronger is the trend to recession. Because of this, from our point of view, accelerated expansion of the Universe takes place; some researchers try to explain it by introducing the dark energy.

\section{Conclusion}

The situation in modern astrophysics is no simple: the motion of planets within the boundaries of the Solar System is well described by the gravity force which is proportional to $1 / R^{2}$, while at much longer distances the speeds of star rotation indirectly point to the fact that the force of their interaction with the center of galaxy is proportional to $1 / R$. Any information about the speeds of the bodies with respect to each other is absent for intermediate distances. In this situation, it is rather difficult to determine which of the approaches (conventionally termed as 1 . The introduction of the dark matter, 2. Modification of Newton's dynamics or 3. Modification of gravity potential) provides a better description of the motion of the matter in the Universe. However, in our opinion, when the dynamics of motion at the periphery of the Solar System will be refined, or if the presence of the DM will be established unambiguously, forcible arguments in favor of one or another approach will appear. The situation may be clarified in future by the American space probes Voyager 1 and Voyager 2 which have already reached the boundaries of the Solar System.

Within the framework of the proposed approach, as a consequence of the domination of the contribution proportional to $1 / \mathrm{R}$ at super long distances in comparison with the contribution proportional to $1 / R^{2}$, there is a definite boundary beyond which the recession of galaxies is favorable from the point of view of energy, which, in particular, does not contradict the accelerated expansion of the Universe.

\section{References}

1. Landay LD, Lifshitz EM (2001) Mechanics (2ndedn) Pergamon Press, Moscow.

2. Oort J H (1932) The force exerted by the stellar system in the direction perpendicular to the galactic plane and some related problems. Bull Astron Inst Netherlands 6: 249.

3. Zwicky F (1933) Die Rotverschiebung von extragalaktischen Nebeln. Helvetica Physica Acta 6: 110-127.

4. Rubin V, Ford W (1970) Rotation of the Andromeda Nebula from a Spectroscopic Survey of Emission Regions. The Astrophysical Journal 159: 379.

5. Ade P A R, Aghanim N, Armitage-Caplan C, et al (2014) Planck 2013 results. I. Overview of products and scientific results. Astronomy and Astrophysics 571: A1 (2014).

6. Milgrom M (1983) A modification of the Newtonian dynamics as a possible alternative to the hidden mass hypothesis. The Astrophysical Journal 270: 365-370.

7. Bekenstein J D (2004). Phys Rev D 70: 8.

8. Brownstein JR, Moffat J W (2007) The Bullet Cluster 1E0657-558 evidence shows modified gravity in the absence of dark matter. Monthly Notices of the Royal Astronomical Society 382: 29-47.

9. Wei Li, Lixin $\mathrm{Xu}$ (2013) Viscous generalized Chaplygin gas as a unified dark fluid Eur Phys J 73: 2471.

10. Hossenfelder S (2008) Bimetric theory with exchange symmetry. Phys Rev D 78: 044015.

11. Kaplan IG (2011) Intermolecular Interactions. John Wiley and Sons. Ltd. Mexico.

12. Roy A (1993) Oxford Illustrated Encyclopedia: The Universe (8thedn) Oxford University Press, UK. 